\title{
PERSONAL SPACE IN VIRTUAL REALITY
}

\author{
Laurie M. Wilcox, Robert S. Allison, Samuel Elfassy and Cynthia Grelik \\ York University, \\ Toronto, Ontario
}

\begin{abstract}
Improving the sense of 'presence' is a common goal of 3D display technology for film, television and virtual reality. However, there are instances in which 3D presentation may elicit unanticipated negative responses. For example, it is well established that violations of interpersonal space cause discomfort in real-world situations. Here we ask if people respond similarly when viewing life-sized stereoscopic images. Observers rated their level of comfort in response to animate and inanimate objects in live and virtual (stereoscopic projection) viewing conditions. Electrodermal activity was also recorded to monitor their physiological response to these stimuli. Subjects exhibited significant negative reactions to violations of interpersonal space in stereoscopic $3 \mathrm{D}$ displays, which were equivalent to those experienced in the natural environment. These data have important implications for the creation of 3D media and the use of virtual reality systems.
\end{abstract}

\section{INTRODUCTION}

Stereoscopic 3D display technology has increasingly been employed for applications such as IMAX ${ }^{\mathrm{TM}}$ theatres and immersive virtual reality systems such as the CAVETM. Considerable research has been directed towards the physiological effects of 3D immersive displays such as fatigue and eye-strain. However, little effort has been made to understand what, if any, effects interpersonal factors have on a viewer's comfort level in immersive environments. One such potential factor is violation of one's interpersonal space. There is considerable evidence in the psychological literature for the existence of an area surrounding a person's body, intrusion into which is often uncomfortable and generally not permitted (Altman, 1975). In the popular sit-com, Seinfeld, a character known as the 'close talker' played on this fact for comic effect. In this paper, we ask whether a virtual close talker would be as bothersome.

Hall (1963) subdivided interpersonal space into four zones. The two of most interest here are the personal distance zone, in which smell, touch, and sight are potentially exchanged, and the social distance zone, in which visual contact is not finely tuned, and smell, touch, and body heat are usually not exchanged (Hall, 1963). Invasions of the personal distance zone elicit the strongest reactions. For instance, Felipe and Sommer (1966) showed that when a violation of personal space occurred by a confederate, participants responded to the intrusion by shifting position, exposing their backs and shoulders to the confederate, or simply leaving the area.

There has been little effort to quantify the effects of violations of interpersonal space in virtual environments. The most relevant work was reported recently by Bailenson, Blascovich, Beall and Loomis (2001). They found that participants tended to keep a greater distance from an avatar (humanlike character) than a similar sized cylinder as they explored a three-dimensional immersive virtual environment. These results are consistent with the hypothesis that personal space is maintained in virtual environments. However, the observed avoidance of the avatar could also be attributed to the fact that it did not appear life-like, and in fact may have been startling. In our first experiment we used 3D images of people and objects presented at a range of viewing distances to determine if observers experience violations of personal space in virtual environments. In the second experiment we directly compared reactions to violations of personal space in live and virtual settings. In this last study, both psychophysical and physiological measures of discomfort were used to assess the impact of intrusions into interpersonal space. 


\section{METHODS}

\section{Subjects}

Participants in Experiments $1(\mathrm{n}=22)$ and $2(\mathrm{n}=16)$ were volunteers ranging in age from 18 to $35 \mathrm{yrs}$. All participants had normal stereopsis as assessed using the RandDot Stereotest TM and wore their prescribed optical correction.

\section{Apparatus and stimuli}

High-resolution stereoscopic images of the stimuli were taken using two Olympus Camedia C-2500L ${ }^{\mathrm{TM}}$ digital cameras fixed at a separation of $6.5 \mathrm{~cm}$. The cameras were calibrated and the images rectified using the Camera Calibration Toolbox for Matlab ${ }^{\mathrm{TM}}$ (www.vision.caltech.edu/bouguetj/calib_doc/index.html). The stimuli were either animate (people) or inanimate (objects). All stimuli were photographed at three distances, 0.5, 1.0, and 2 meters.

In the virtual test sessions, images were presented using an Electrohome Model Marquee-8000 CRT projector and a 2.35 by 1.73 meter back-projection screen. All testing was performed in a dimly lit room; illumination and room temperature were held constant to avoid potential confounds (Adams and Zuckerman, 1991). The observers were seated in a chair $1 \mathrm{~m}$ from the screen with their heads positioned in a chin rest. The subjects wore Stereographics ${ }^{\mathrm{TM}}$ 3D shutter glasses that switched in synchrony with the projector (at $120 \mathrm{~Hz}$, giving $60 \mathrm{~Hz}$ per eye). This allowed presentation of the appropriate stereoscopic half-images to each eye separately. A Macintosh G3 computer and MATLAB ${ }^{\mathrm{TM}}$ software were used to present the stimuli, and to record the verbal ratings made by the subjects (input by the experimenter).

In Experiment 2, in the live test condition, a chin rest was also used and a mechanical shutter controlled the participants' view. Objects were mounted on pedestals so that the vertical centre of the object was at the eye level of the participant. The animate stimuli were seated facing the observer, again at eye level. As in the 3D images of these same people, the animate stimuli fixated on a point 20 degrees to the right of the observer.

A physiological measure of arousal was taken in Experiment 2, in both the live and virtual test conditions. Subject's electrodermal activity (EDA) was monitored using a Beckman GSR Type R411 module in constant current mode. The output of the module was connected to the experimental computer via a National Instruments PCI-MIO-16XE-50 data acquisition card sampling at $20 \mathrm{~Hz}$ with 16-bit precision. The device was calibrated prior to each session using fixed impedance calibration points so that voltages on the data acquisition card could be converted to skin conductance values. At the beginning of each experimental session, $\mathrm{Ag} / \mathrm{AgCl}$ surface electrodes were connected to the medial phalanx of the index and middle finger of the participant's non-dominant hand using an isotonic paste. Following application of the electrodes, the interface was given time to stabilize and a baseline EDA reading was taken for 120 seconds. For each trial, continuous recordings of EDA data began 2 seconds prior to presentation of the stimulus and ended when the stimulus disappeared.

\section{Procedure}

Participants viewed each image once (to avoid familiarity effects) in quasi-random order. In Experiment 1 images were viewed for 5s. In Experiment 2 each trial lasted 12 s, to permit adequate EDA sampling, and in the live test condition to provide sufficient time to exchange the stimuli. In all conditions, when the test image/object was removed participants were asked to rate their level of comfort in response to the stimulus they had just viewed. A Likert scale was used, which ranged from 1 (comfortable, not intrusive) to 7 (very uncomfortable, very intrusive). In Experiment 1 the observers participated in the virtual condition only, while in Experiment 2 each participant was tested in a live and a virtual session, on non-consecutive days. To avoid order effects, half of the participants in Experiment 2 were tested with live stimuli first, virtual second and vice versa.

\section{RESULTS}

\section{Experiment 1}

Figure 1 shows the average rating data for stimuli presented at each of the three distances in Experiment 1 (virtual testing only). 


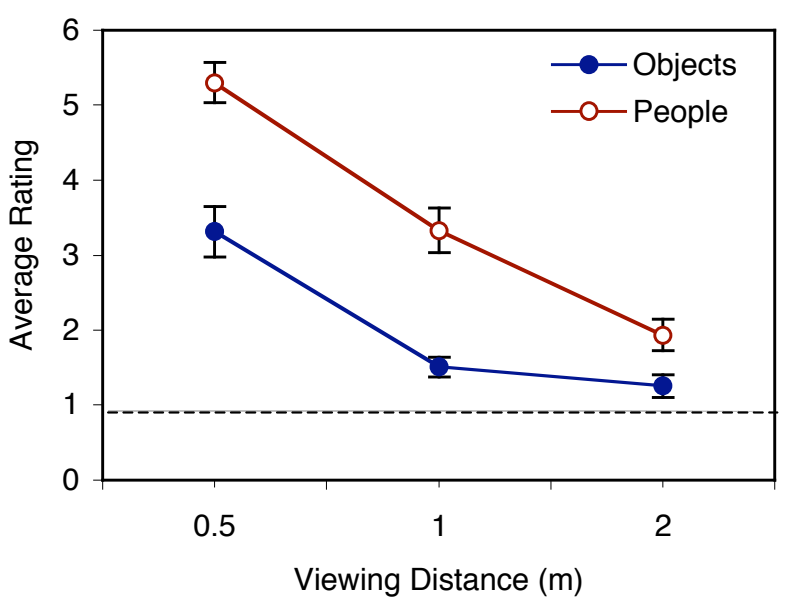

Figure 1. Comfort ratings obtained in response to $3 \mathrm{D}$ images of people (open circles) and objects (closed circles) at each of three viewing distances. Ratings are averaged across 22 subjects, and the error bars represent \pm one standard error of the mean.

A repeated-measures ANOVA showed main effects of both viewing distance $(\mathrm{F}(1.1,23.3)=132.35, \mathrm{p}<$ $0.05)$ and stimulus type $(\mathrm{F}=(1,21)=179.40, \mathrm{p}<$ $0.05)$. The interaction between these two variables was also significant $(\mathrm{F}(1.29,27.21)=15.96, \mathrm{p}<0.05)$. Note that the assumption of sphericity was violated for the distance and interaction analyses so the Greenhouse-Geisser correction was applied. From this analysis and Figure 1 it is clear that there is an increase in discomfort as 3D images are moved closer to the observer. Further, the reaction to these images is more intense when the images depict people rather than objects. The proximity effect is more pronounced for animate stimuli as demonstrated by a significant interaction between distance and object type.

\section{Experiment 2}

Experiment 1 showed that individuals do experience violations of interpersonal space when presented with virtual 3D images of people and objects. Further, both this proximity effect and the mean discomfort level were higher for people than for objects. In Experiment 2 we make a direct comparison between the identical animate and inanimate stimuli presented 'live' or virtually. Further, in this study we include a measure of physiological arousal to corroborate observers' subjective ratings.

\section{Rating Results}

Figure 2 depicts rating data from Experiment 2 for the virtual (A) and live (B) test conditions.

$2 \mathrm{~A}$

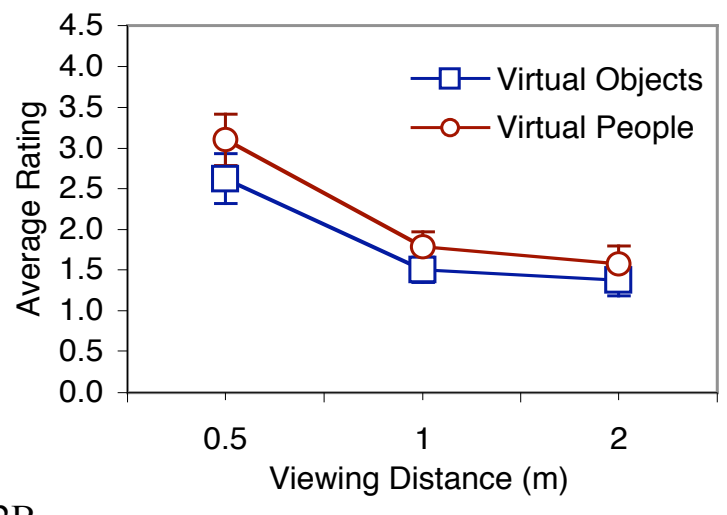

2B

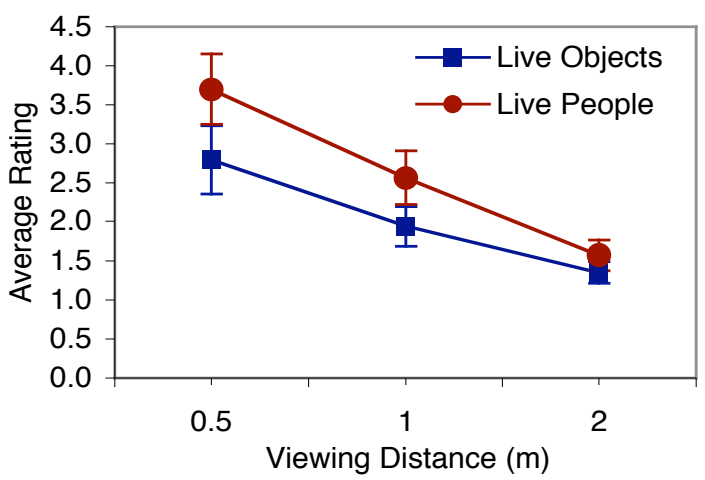

Figure 2 (A and B). Average rating data are shown here for objects (squares) and people (circles) in the virtual (A) and live (B) test conditions. Data are averaged across 16 subjects, and the error bars represent \pm one standard error of the mean.

As in Experiment 1, animate stimuli are consistently rated higher (more uncomfortable) than inanimate stimuli and discomfort increases as the stimuli approach the observers. Results of the analysis of variance showed main effects of both viewing distance $(\mathrm{F}(1.4,21.6)=41.8, \mathrm{p}<.05)$, and stimulus type $(\mathrm{F}(1,15)=5.99 \mathrm{p}<.05)$. The GreenhouseGeisser correction was implemented for the evaluation of the effect of distance. There was no difference between the ratings made in the live and virtual test conditions $(\mathrm{F}(1,15)=2.9, \mathrm{p}>.05)$ and none of the remaining interaction terms were significant. Closer evaluation of these data revealed six subjects whose pattern of responses was inconsistent with that reported in Experiment 1; that 
is they did not show the classic proximity effect. We repeated the analysis of variance, including only those observers who showed the proximity effect in the live test condition. The rationale is that it is unreasonable to expect observers who do not experience the phenomenon in real exposure conditions, to exhibit it in response to 3D images. This analysis $(n=10)$ also shows significant main effects of viewing distance, and of stimulus type. Importantly, when individuals who do not exhibit the proximity effect in the live test condition are removed, the results replicate those of the first Experiment. In particular there was a significant stimulus by distance interaction $(\mathrm{F}(1.2,10.8)=15.8$, $\mathrm{p}<.05)$ that was not present in the original analysis. As noted below, removal of these subjects did not effect the pattern of the EDA data.

\section{EDA Results}

EDA was quantified as the difference between the between skin conductance prior to stimulus presentation and the peak reading during a trial. Figure 3 shows the average reading for each image class at each distance for both live (A) and virtual (B) settings.
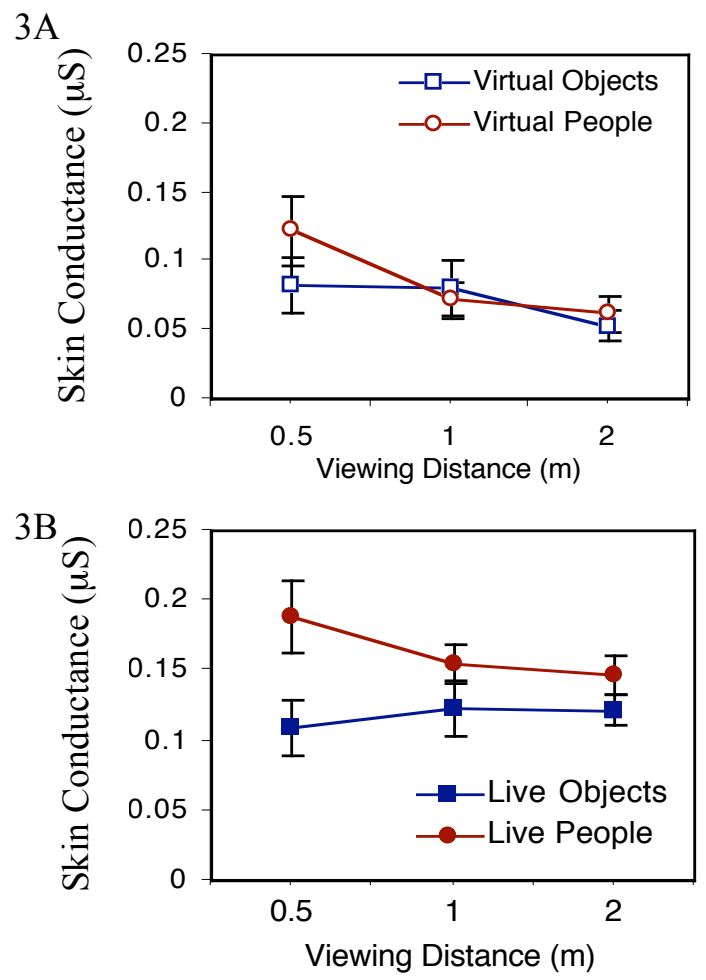

Figure 3 (A and B). Average EDA data are shown here for objects (squares) and people (circles) in the virtual (A) and live (B) test conditions. Data are averaged across 12 subjects, and the error bars represent \pm one standard error of the mean.

Due to technical difficulties with the recording apparatus EDA data from four of the subjects were not included $(\mathrm{n}=12)$. Analysis of variance (using the Greenhouse-Geisser correction) of the EDA results revealed a significant interaction between the type of stimulus and viewing distance $(\mathrm{F}(1.53,16.9)$ $=4.1, \mathrm{p}<0.05)$. The identical pattern of results was obtained when those who did not experience the proximity effect in the live test conditions were omitted $(n=9)$.

\section{DISCUSSION}

We have shown that intrusion into one's personal distance zone (Hall, 1963) by virtual 3D stimuli can cause significant negative reactions. As predicted, this proximity effect is greater for images of people than for images of objects, and decreases at viewing distances greater than $0.5 \mathrm{~m}$. Experiment 2 demonstrated that the response to invasion of virtual personal space is equivalent to the response to the same stimuli in a live setting. Further, physiological measures of arousal are consistent with the observers' ratings, a finding that corroborates our subjective rating measure.

Comparison of Experiments 1 and 2 shows larger differences between ratings for animate and inanimate stimuli in the first study. This is likely due to the subject population. That is, naïve students were recruited for Experiment 1, most of whom had never been in an experimental setting before. On the other hand in Experiment 2, because subjects were required to participate in two sessions, we recruited subjects from neighbouring vision laboratories. The latter group had no information regarding the nature of the experiment, but most had participated in psychophysical studies previously. As a result they were generally more comfortable and tended to make lower ratings. This difference likely explains why several subjects in Experiment 2 failed to exhibit the classic proximity effect, even under live test conditions.

One of the objectives of Experiment 2 was to make a direct comparison of subject's reaction to live and virtual stimuli at different viewing distances. To this end we calculated the ratio of ratings in the live and virtual test conditions for 
objects $(1.1,1.29,0.98$ for $0.5,1$ and $2 \mathrm{~m}$

respectively) and people $(1.19,1.43,1.0)$. Ratios

equal to one indicate that the ratings were identical, while ratios greater than one result from lower ratings in the virtual condition.

It is clear that ratings were very similar, particularly at the near and far viewing distances. Further, the pattern of ratings was the same for objects and people. That is in both cases, the live and virtual ratings were most similar at viewing distances of 0.5 and $2 \mathrm{~m}$. At $1 \mathrm{~m}$ ratings in response to virtual stimuli were lower than responses to live stimuli. It is possible that this is related to the reduction in cue conflict at $1 \mathrm{~m}$. That is, the stereoscopic images were taken at three viewing distances, however, the observers were always seated $1 \mathrm{~m}$ from the display. Therefore, in the $1 \mathrm{~m}$ condition, there was less conflict between vergence and accommodation. It is important to note that discomfort introduced by the display characteristics cannot account for our proximity results; such effects should have applied equally to animate and inanimate stimuli.

Lombard (1995) has proposed an evolutionary explanation for 'parasocial' relationships. He has argued that humans have not had sufficient exposure to audio-visual media to allow them to adapt their social responses. Therefore, stimuli presented in immersive environments are not correctly processed as symbols, and as a result elicit the same responses as in live interactions. The use of stereoscopic displays is an important component of this response, for photographs of people do not elicit avoidance behaviour (Argyle and Dean, 1965). Thus, while an improved sense of presence may result from the use of 3D display technology, this presence elicits both positive and negative aspects of interactions in the real world. Therefore, the content and the intended audience impact should be jointly considered when choosing display media. For instance, if the goal is a positive, relaxed reaction to animate stimuli, then
3D displays should be used with a moderate range of depth values. However, this phenomenon can also be exploited to, say, evoke real audience discomfort when faced with a virtual 'closetalker'.

\section{REFERENCES}

Adams, L. \& Zuckerman, D. (1991). The effect of lighting conditions on personal space requirements. The Journal of General Psychology, 118(4), 335-340.

Altman, I. (1975). The environment and social behaviour: Privacy, personal space, territory, crowding. Monterey, CA: Brooks/Cole Publishing Company.

Argyle, M. \& Dean, J. (1965). Eye-contact, distance and affiliation. Sociometry, 28(3), 289-304.

Bailenson J.N., Blascovich J., Beall A.C., and Loomis J. (2001) Equilibrium Theory Revisited: Mutual Gaze and Personal Space in Virtual Environments. Presence: Teleoperators and Virtual Environments, 10, (6), 583 - 598.

Felipe, N. J. \& Sommer, R. (1966). Invasions of personal space. Social Problems, 14(2), 206-214.

Hall, E. T. (1963) A system for the notation of proxemic behavior. American Anthropologist, 65, 1003-1026.

Hayduk, L. A. (1981). The shape of personal space An experimental investigation. Canadian Journal Of Behavioural Science, 13(1), 87-93.

Lombard, M. (1995). Direct responses to people on the screen: Television and personal Space. Communications Research, 22(3), 288-32 